\title{
Frank's constant in the hexatic phase
}

\author{
P. Keim, G. Maret*, and H.H. von Grünberg \\ Universität Graz, 8010 Graz, Austria \\ *Universität Konstanz, 78457 Konstanz, Germany
}

(Dated: August 8, 2018)

\begin{abstract}
Using video-microscopy data of a two-dimensional colloidal system the bond-order correlation function $G_{6}$ is calculated and used to determine the temperature-dependence of both the orientational correlation length $\xi_{6}$ in the isotropic liquid phase and the Frank constant $F_{A}$ in the hexatic phase. $F_{A}$ takes the value $72 / \pi$ at the hexatic $\leftrightarrow$ isotropic liquid phase transition and diverges at the hexatic $\leftrightarrow$ crystal transition as predicted by the KTHNY-theory. This is a quantitative test of the mechanism of breaking the orientational symmetry by disclination unbinding.
\end{abstract}

PACS numbers: $64.70 . \mathrm{Dv}, 68.35 . \mathrm{Rh}, 82.70 . \mathrm{Dd}$

The theory of melting in two dimensions (2d) developed by Kosterlitz, Thouless, Halperin, Nelson and Young (KTHNY-theory) suggests a two-stage melting from the crystalline phase to the isotropic liquid. The first transition at temperature $T_{m}$ is driven by the dissociation of thermally activated dislocation pairs into isolated dislocations breaking the translational symmetry 1, 2]. The fluid phase directly above $T_{m}$ still exhibits orientational symmetry and is called the hexatic phase. It may be viewed as an anisotropic fluid with a six-fold director 3, 4] which is characterized by a finite value of Frank's constant $F_{A}$, the elastic modulus quantifying the orientational stiffness. At the second transition at $T_{i}>T_{m}$, the dissociation of some of the dislocations into free disclinations destroys the orientational symmetry. Now, the fluid shows ordinary short-range rotational and positional order as it is characteristic of an isotropic liquid.

Following an argument given in [1, 4], $T_{m}$ and $T_{i}$ can be estimated using the defect interaction Hamiltonian $H_{d}$ between a pair of disclinations $(d=d i s c)$ and a pair of dislocations $(d=d i s l)$ which for both defect pairs and at large distances goes like $H_{d} \sim c_{d} \log r$ with the dimensionless strength parameter $c_{d}$ depending on the defect type. Defect dissociation is completed at a temperature where the thermally averaged pair distance $\left\langle r_{d}^{2}\right\rangle$ diverges. Evaluating this expression for $H_{d}$ one generally finds divergence if $c_{d}=4$. The unbinding condition $c_{d}=4$ translates into $\lim _{T \rightarrow T_{m}^{-}} \beta \mathcal{K}(T) a_{0}^{2}=16 \pi$ for dislocation pairs ( $\beta=1 / k_{B} T, a_{0}$ is lattice spacing) and into $\lim _{T \rightarrow T_{i}^{-}} \beta F_{A}(T)=72 / \pi$ for disclination pairs, where $\mathcal{K}$ is the Young's modulus of the crystal. Connecting thus the defect pair unbinding condition to the two transition temperatures $T_{i}$ and $T_{m}$, two expressions are obtained that summarize the microscopic explanation of the KTHNY theory for two-stage melting.

In this Letter we study the temperature-dependence of Frank's constant of a 2D system in the hexatic phase. We first determine the hexatic $\rightarrow$ isotropic fluid transition temperature $T_{i}$ and then check if Frank's constant takes the value $72 / \pi$ at $T_{i}$, thus testing the KTHNY theory and its prediction that disclination unbinding occurs at $T_{i}$. In addition, we analyze the divergence behavior of the orientational correlation length at $T_{i}$ and of Frank's constant at $T_{m}$.

Different theoretical approaches invoking grain boundary induced melting [5, 6] or condensation of geometrical defects [7, 8] suggest one first order transition. However some simulations for Lennard-Jones systems indicate the hexatic phase to be metastable [9, 10. The transition in hard-core systems seem to be first-order 12 . probably due to finite-size effects 11]. Simulations with long-range dipole-dipole interaction clearly show second order behavior [13. Experimental evidence for the hexatic phase has been demonstrated for colloidal systems 14, 15, 16, 17, 18, 19], in block copolymer films 20, 21], as well as for magnetic bubble arrays and macroscopic granular or atomic systems 22, 23, 24, 25, 26]. Still the order of the transitions is seen to be inconsistent. The observation of a phase equilibrium isotropic/hexatic 17, 21 and hexatic/crystalline 17] indicates two first order transitions. In our system we find two continuous transitions.

The experimental setup is essentially the same as in 27]. Spherical and super-paramagnetic colloids (diameter $d=4.5 \mu \mathrm{m}$ ) are confined by gravity to a water/air interface formed by a water drop suspended by surface tension in a top sealed cylindrical hole of a glass plate. The field of view has a size of $835 \times 620 \mu \mathrm{m}^{2}$ containing typically up to $3 \cdot 10^{3}$ particles (out of $3 * 10^{5}$ of the whole sample). A magnetic field $\vec{H}$ is applied perpendicular to the air/water interface inducing in each particle a magnetic moment $\vec{M}=\chi \vec{H}$. This leads to a repulsive dipole-dipole pair-interaction with the dimensionless interaction strength given by $\Gamma=\beta\left(\mu_{0} / 4 \pi\right)(\chi H)^{2}(\pi \rho)^{3 / 2}$. Here $\chi$ is the susceptibility per colloid while $\rho$ is the $2 \mathrm{~d}$ particle density and the average particle distance is $a=1 / \sqrt{\rho}$. The interaction strength can be externally controlled by means of the magnetic field $H$; it can be interpreted as an inverse temperature and is the only parameter controlling the phase behavior of the system. For each $\Gamma$ the coordinates of the colloids are recorded 


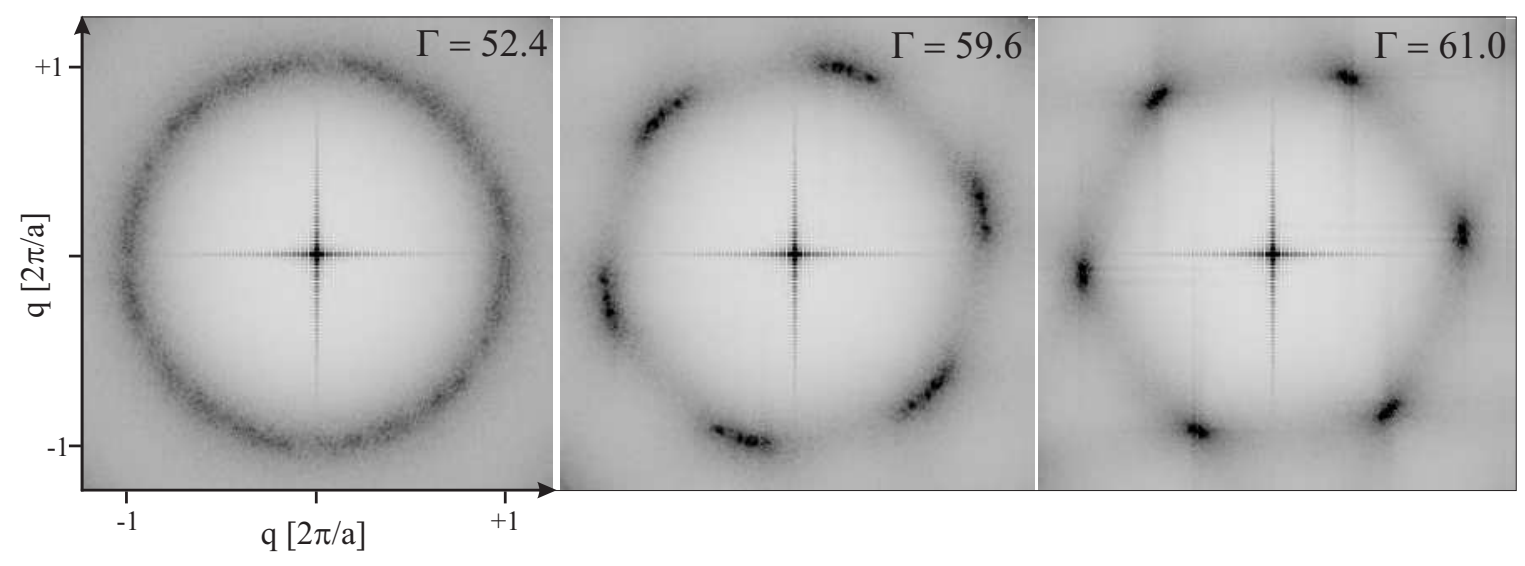

FIG. 1: Structure factor $S(\vec{q})$ of our colloidal system at three different inverse temperatures $\Gamma$ corresponding to the isotropic liquid $(\Gamma=52.4)$, the hexatic phase $(\Gamma=59.6)$ and the crystalline $(\Gamma=61.0)$ phase. The central cross is an artifact of the Fourier-transformation.

via video-microscopy (resolution of particle position $d r=100 \mathrm{~nm}$ ) and digital image processing over a period of $1-2 h$ using a frame rate of $250 \mathrm{~ms}$.

To set the stage we first visualize in Fig. (11) the three phases and their symmetries by plotting the structure factor

$$
S(\vec{q})=\frac{1}{N}\left\langle\sum_{\alpha, \alpha^{\prime}} e^{-i \vec{q}\left(\vec{r}_{\alpha}-\vec{r}_{\alpha^{\prime}}\right)}\right\rangle
$$

as calculated from the positional data of the colloids for three different temperatures. Here, $\alpha, \alpha^{\prime}$ runs over all $N$ particles in the field of view while \langle\rangle denote the time average over 700 configurations. In the liquid phase, concentric rings appear having radii that can be connected to typical inter-particle distances. The hexatic phase, on the other hand, is characterized by six segments of a ring which arise due to the quasi long-range orientational order of the six-fold director [28]. In the crystalline phase the Bragg peaks of a hexagonal crystal show up with a finite width that is due to the quasi long-range character of the translational order.

To quantify the six-fold orientational symmetry the bond-order correlation function

$$
G_{6}(r)=\left\langle\psi(\vec{r}) \psi^{*}(\overrightarrow{0})\right\rangle
$$

is calculated with $\psi(\vec{r})=\frac{1}{N_{j}} \sum_{j} e^{6 i \theta_{i j}(\vec{r})}$. Here the sum runs over the $N_{j}$ next neighbors of the particle $i$ at position $\vec{r}$ and $\theta_{i j}(\vec{r})$ is the angle between a fixed reference axis and the bond of the particle $i$ and its neighbor $j$. \langle\rangle here denotes not only the ensemble average which is taken over all $N(N-1) / 2$ particle-pair distances for each configuration (resolution $d r=100 \mathrm{~nm}$ ) but also the time average over 70 statistically independent configurations.
KTHNY theory predicts that

$$
\begin{aligned}
\lim _{r \rightarrow \infty} G_{6}(r) & \neq 0 & & \text { crystal: long range order } \\
G_{6}(r) & \sim r^{-\eta_{6}} & & \text { hexatic: quasi long range } \\
G_{6}(r) & \sim e^{-r / \xi_{6}} & & \text { isotropic: short range }
\end{aligned}
$$

$\eta_{6}<1 / 4$ and takes the value $1 / 4$ right at $T=T_{i}$. All three regimes can be easily distinguished in Fig. (2) showing $G_{6}(r)$ for a few representative temperatures. Note, that $G_{6}(0)$ is not normalized to 1.

We next fit $G_{6}(r)$ to $r^{-\eta_{6}}$ and $e^{-r / \xi_{6}}$ to extract $\eta_{6}$ and $\xi_{6}$. The fits are performed for radii $r / a \in\{0 . .20\}$ [29]. To check for the characteristics of the orientational correlation function, the ratio of the reduced chi-square

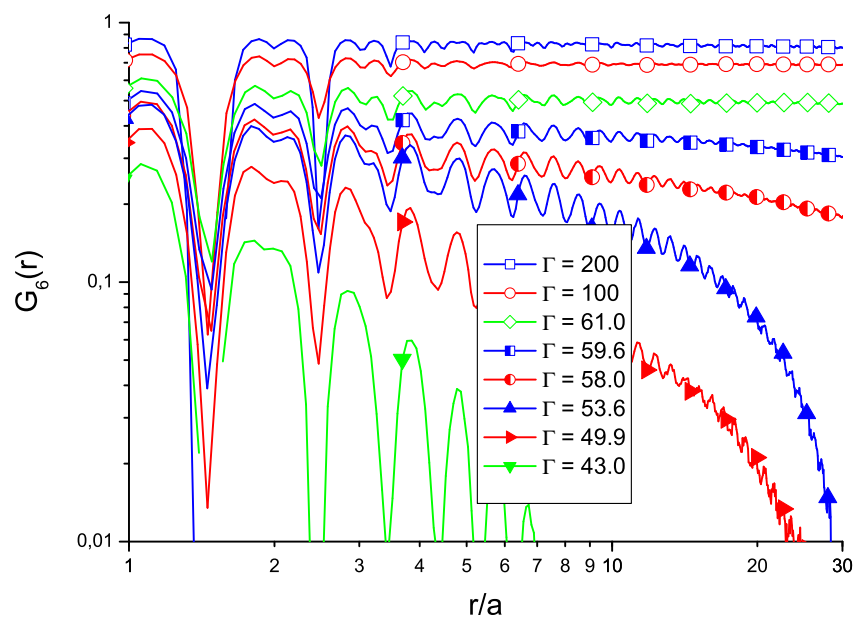

FIG. 2: Orientational correlation function $G_{6}(r)$ as function of the inverse temperature $\Gamma$ in a log-log plot. From top to bottom: Three curves for the crystalline phase showing the long-range orientational order $\left(\lim _{r \rightarrow \infty} G_{6}(r) \neq 0\right)$, two curves showing the quasi long-range order of the hexatic phase $\left(G_{6}(r) \sim r^{-\eta_{6}(\Gamma)}\right)$ and three curves showing the short-range order typical of the isotropic liquid $\left(G_{6}(r) \sim e^{-r / \xi_{6}(\Gamma)}\right)$. 


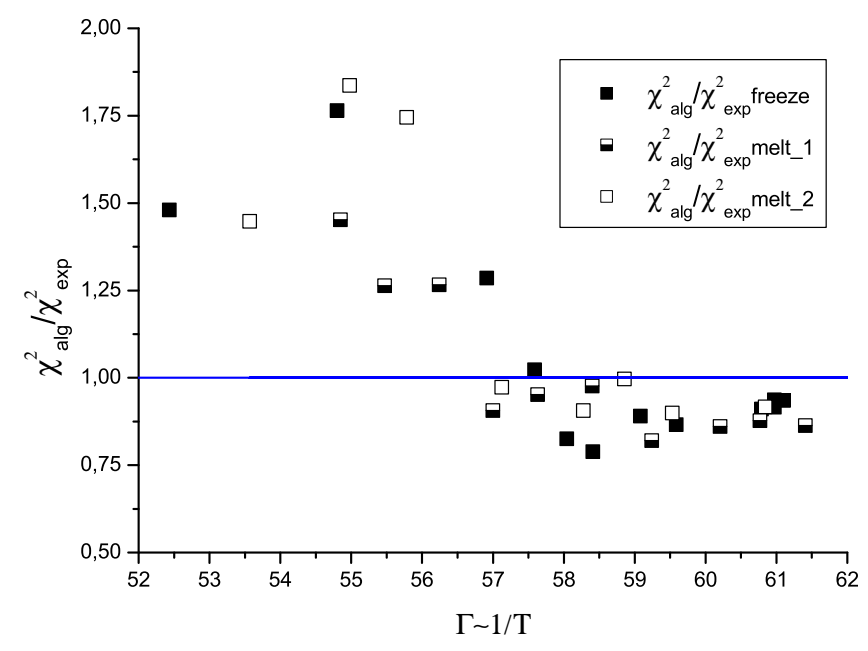

FIG. 3: Quantitative test for the long distance behavior of $G_{6}(r)$. For $\chi_{a l g}^{2} / \chi_{e x p}^{2}<1$ the algebraic decay fits better.

$\chi^{2}$ goodness-of-fit statistic of the algebraic $\left(\chi_{\text {alg }}^{2}\right)$ and exponential $\left(\chi_{\text {exp }}^{2}\right)$ fit is shown in Fig. (3) as a function of $\Gamma$ for three different measurements. For melting, a crystal free of dislocations was grown at high $\Gamma$ and then $\Gamma$ was reduced in small steps. For each temperature step the system was equilibrated $1 / 2 h$ before data acquisition started. This was done at different densities: melt_1 with average particle distance of $a=11.8 \mu \mathrm{m}$ and melt_2 with $a=14.8 \mu \mathrm{m}$ containing 3200 respectively 2000 particles in the field of view. The measurement denoted freeze in Fig. (3) $(a=11.8 \mu \mathrm{m})$ started in the isotropic liquid phase and $\Gamma$ was increased with an equilibration time of $1 h$ between the steps. For $\chi_{\text {alg }}^{2} / \chi_{\text {exp }}^{2}>1$ an exponential decay fits better than the algebraic and vice versa for $\chi_{\text {alg }}^{2} / \chi_{\text {exp }}^{2}<1$. We observe in Fig. (3) that the change in the characteristic appears at $\Gamma_{i}=57.5 \pm 0.5$. This value is the temperature of the hexatic $\leftrightarrow$ isotropic liquid transition.

In the vicinity of the phase transition, approaching $\Gamma_{i}$ from the isotropic liquid the orientational correlation length $\xi_{6}$ should diverge as [4],

$$
\xi_{6}(\Gamma) \sim \exp \left(\frac{b}{\left|1 / \Gamma-1 / \Gamma_{i}\right|^{\nu}}\right),
$$

with $b$ a constant and $\nu=1 / 2$. This behavior is observed in Fig. (4 $). \xi_{6}$ indeed increases dramatically near $\Gamma_{i}=$ $57.5 \pm 0.5$ irrespective of whether the system is heated or cooled. Before discussing this feature we first address the finite size effect. To this end, we have computed $G_{6}(r)$ and $\xi_{6}$ for subsystems of different size ranging from $720 \times$ $515 \mu \mathrm{m}^{2}, 615 \times 405 \mu \mathrm{m}^{2}, 505 \times 300 \mu \mathrm{m}^{2}, 400 \times 190 \mu \mathrm{m}^{2}$ to $390 \times 80 \mu \mathrm{m}^{2}$. The resulting data-points are plotted as triangles in Fig. (4) and belong to the black filled squares which they converge to. No finite size effect is found for $\Gamma<56$, but a considerable one at $\Gamma=56.9$ close to $\Gamma_{i}$ where we obviously need the full field of view to capture the characteristic of the divergence. At $\Gamma=58.0$ there is a huge finite size effect indicating that $\xi_{6}$ is much larger than the field of view. However, inside the hexatic phase, $\xi_{6}$ is no longer well defined as the decay is algebraic. We fit our data to eq. (3) in the range $49<\Gamma<57.5$ and find the critical exponent $\nu=0.5 \pm 0.03$ and $\Gamma_{i}=58.9 \pm 1.1$, a value which due to the finite-size effect is slightly larger than $\Gamma_{i}$ obtained from Fig. (3).

The exponent $\eta_{6}$ is related to Frank's constant $F_{A}$ [4]

$$
\eta_{6}(\Gamma)=\frac{18 k_{B} T}{\pi F_{A}(\Gamma)}
$$

So the critical exponent $\eta_{6}\left(\Gamma_{i}\right)=1 / 4$ corresponds to $\beta F_{A}\left(\Gamma_{i}\right)=72 / \pi$ at the hexatic $\leftrightarrow$ liquid transition. This quantity is plotted in Fig. (40). Indeed, $F_{A}$ crosses the value $72 / \pi$ at $\Gamma_{i}=57.5 \pm 0.5$ exactly at that temperature which in Fig. (3) has been independently determined to


FIG. 4: Correlation length $\xi_{6}$ (a) and Frank constant $F_{A}$ (b) as a function of the inverse temperature. $\xi_{6}$ diverges at $\Gamma_{i}$ and $F_{A}$ at $\Gamma_{m}$. In between the system shows hexatic symmetry. The solid lines are fits to eq. (3) and (5) resulting in critical exponents $\nu=0.5 \pm 0.03$ and $\bar{\nu}=0.35 \pm 0.02$ respectively. Triangles are shifted by $0.1 \Gamma$ for clarity. 
be the transition temperature $T_{i}$. For $\Gamma<\Gamma_{i}, F_{A}$ should jump to zero which is not completely reproduced. We note that since $\eta_{6}$ is not well defined in the isotropic fluid, it becomes problematic to extract $F_{A}$ from eqn. (4) below $\Gamma_{i}$. At $\Gamma_{m}$, at the hexatic $\rightarrow$ crystalline transition, $F_{A}$ must diverge which indeed it does. This divergence can be identified with the divergence of the square of the translational correlation length $\xi_{+}$[4]

$$
F_{A}(\Gamma) / k_{B} T \sim \xi_{+}^{2} \sim \exp \left(\frac{2 c}{\left|1 / \Gamma-1 / \Gamma_{m}\right|^{\bar{\nu}}}\right)
$$

where $c$ is again a constant and $\bar{\nu}=0.36963$. Fitting the values of $F_{A}$ to the expression in eqn. (5) in the range $57.5<\Gamma<61$ we obtain $\bar{\nu}=0.35 \pm 0.02$ and $\Gamma_{m}=61.3 \pm 0.4$ as an upper threshold. Again triangles represent evaluation of our data in sub-windows of variable size (same sizes as above). The finite size effect for $\Gamma=57.0$ is negligible. Close to $\Gamma_{m}$ it increases but the values saturates for $\Gamma=59.1$ and $\Gamma=60.8$ and remain within the error-bars for the biggest sub-windows.

In conclusion, we have checked quantitatively the change of quasi-long-range to short-range orientational order and extracted the correlation length $\xi_{6}$ in the isotropic fluid and Frank's constant $F_{A}$ in the hexatic phase from trajectories of a $2 \mathrm{~d}$ colloidal system. We find a hexatic $\leftrightarrow$ isotropic liquid transition at $\Gamma_{i}=57.5 \pm 0.5$. Three observations support this result: (i) the change of the distance dependence of $G_{6}(r)$ (Fig. (3)), (ii) the condition $F_{A}\left(\Gamma_{i}\right)=72 / \pi$ for Frank's constant and (iii) the divergence of $\xi_{6}$. For the transition hexatic $\leftrightarrow$ crystal $F_{A}$ diverges at $\Gamma_{m}$. Both divergencies (extracted from just one correlation function) lead to critical exponents that are in good agreement with the KTHNY-theory. The measurements for melting and freezing support each other; so we may conclude that there is no hysteresis effect of the phase-transitions. At the two transitions, the order parameters are observed to change continuously (within the resolution of $\Gamma \propto 1 / T$ ); no indication of a phase-separation (as for example strong fluctuations of the order parameters) has been found [30] as has been reported by $[17,21]$. So we believe that in our system having a well-defined, purely repulsive pair-potential and a confinement to $2 \mathrm{D}$ that is free of any surface roughness - the transitions are second order.

In [31, 32] we verified that the Young's modulus becomes $16 \pi$ at $T_{m}$. We have now checked that $F_{A}$ takes the value $72 / \pi$ at $T_{i}$. These two findings together confirm the two-stage KTHNY melting scenario with its underlying microscopic picture of breaking the translational symmetry by dislocation-pair- and orientational symmetry by disclination-pair-unbinding.

P. Keim gratefully acknowledges the financial support of the Deutsche Forschungsgemeinschaft.
[1] J. Kosterlitz and D. Thouless, J. Phys. C, 6, 1181 (1973).

[2] A.P. Young, Phys. Rev. B, 19, 1855 (1979).

[3] B.I. Halperin and D.R. Nelson, Phys. Rev. Lett. 41, 121 (1978).

[4] D.R. Nelson and B.I. Halperin, Phys. Rev. B 19, 2457 (1979).

[5] S.T. Chui, Phys. Rev. B 28, 178 (1983).

[6] H. Kleinert, Phys. Lett. 95A, 381n (1983).

[7] M.A. Glaser and N.A. Clark, Adv. Chem. Phys.83, 543 (1993).

[8] Y. Lansac, M.A. Glaser, and N.A. Clark, Phys. Rev. E 73, 041501 (2006).

[9] K. Chen, T. Kaplan, and M. Mostoller, Phys. Rev. Lett. 74, 4019 (1995).

[10] F.L. Somer, G.S. Canright, T. Kaplan, K. Chen, and M. Mostoller, Phys. Rev. Lett. 79, 3431 (1997).

[11] C.H. Mak, Phys. Rev. E, 73, 065104(R) (2006).

[12] A. Jaster, Phys. Rev. E 59, 2594 (1999).

[13] S.Z. Lin, B. Zheng, and S. Trimper, Phys. Rev. E. 73, 066106 (2006).

[14] C.A. Murray and D.H. Van Winkle, Phys. Rev. Lett, 58, 1200 (1987).

[15] Y. Tang, A.J. Armstrong, R.C. Mockler, and W.J. O'Sullivan, Phys. Rev. Lett, 62, 2401 (1989).

[16] R.E. Kusner, J.A. Mann, J. Kerins, and A.J. Dahm, Phys. Rev. Lett, 73, 3113 (1994).

[17] A.H. Marcus and S.A. Rice, Phys. Rev. Lett, 77, 2577 (1996).

[18] K. Zahn and G. Maret, Phys. Rev. Lett, 85, 3656 (2000).

[19] A.V. Petukhov, D. van der Beek, R.P.A. Dullens, I.P. Dolbnya, G.J. Vroege, and H.N.W. Lekkerkerker, Phys. Rev. Lett, 95, 077801 (2005).

[20] R.A. Segalman, A. Hexemer, R.C. Hayward, and E.J. Kramer, Macromolecules, 36, 3272 (2003).

[21] D.E. Angelescu, C.K. Harrison, M.L. Trawick, R.A. Register, P.M. Chaikin, Phys. Rev. Lett. 95, 025702 (2005).

[22] R. Seshadri and R.M. Westervelt, Phys. Rev. B. 46, 5142 (1992).

[23] P.M. Reis, R.A. Ingale, and M.D. Shattuck, Phys. Rev. Lett. 96, 258001 (2006).

[24] X.H. Zheng and R. Grieve, Phys. Rev. B. 73, 064205 (2006).

[25] P. Dimon, P.M. Horn, M. Sutton, R.J. Birgeneau, and D.E. Moncton, Phys. Rev. B, 31, 437 (1985).

[26] D. Li and S.A. Rice, Phys. Rev. E. 72, 041506 (2005).

[27] P. Keim, G. Maret, U. Herz, and H.H. von Grünberg, Phys. Rev. Lett. 92, 215504 (2004).

[28] The segments will merge to rings, if the systems size tends towards infinity.

[29] The upper value is motivated by the maximum value of the histogram of distances and $r / a=0$ is excluded in the algebraic case to avoid the singularity. The histrogram over distances is used as statistical weight of the fit-function taking into account the different frequency of occurrence of the data-points in the minima and maxima of $G_{6}(r)$.

[30] We indeed do see a local clustering of dislocations as already noted by [14, 15] in the hexatic phase close to $\Gamma_{i}$ (which is not too surprising for dislocations with finite density having an attractive interaction) but this averages out in $G_{6}$ if the field of view is big enough. 
[31] H.H. von Grünberg, P. Keim, K. Zahn, and G. Maret, Phys. Rev. Lett. 93, 255703 (2004).

[32] J. Zanghellini, P. Keim, and H.H. von Grünberg, J. Phys.
Cond. Mat. 17, 3579 (2005). 